\title{
BulK Binding update PRocedure For PMIPV6 BASED INTELLIGENT TRANSPORTATION SYSTEMS
}

\author{
$\mathrm{K} \mathrm{Vasu}^{1}$, Sudipta Mahapatra ${ }^{1}$ and C S Kumar ${ }^{2}$ \\ ${ }^{1}$ Department of E\&ECE, IIT Kharagpur, Kharagpur, West Bengal, India \\ vasukanster@gmail.com \\ sudipta@ece.iitkgp.ernet.in \\ ${ }^{2}$ Department of Mechanical Engineering, \\ IIT Kharagpur, Kharagpur, West Bengal, India \\ kumaramech.iitkgp.ernet.in
}

\begin{abstract}
Intelligent transportation system (ITS) consists of moving networks, where the network mobility (NEMO) basic support is adopted as a mobility management protocol for moving networks. Even though NEMO basic support (NBS) provides a basic mobility support for ITS systems, the mobile routers $(M R)$ need to participate in the mobility signaling. In the literature, network based mobility management such as Proxy Mobile IPv6 (PMIPv6) based solutions are explored for mobility management. However, the signaling overhead incurred due to this approach is still need to be optimized. In this paper, we introduce a bulk binding update solution for the registration of MR with local mobility anchor (LMA) in moving networks. The bulk binding update procedure uses a group identifier for group of MRs during the periodic binding update process which reduces the signaling overhead compared with the basic PMIPv6 based approach. The numerical results demonstrate that the proposed approach gives a better performance in terms of signaling overhead and handover latency than NBS, and simple PIMPv6 based solutions.
\end{abstract}

\section{KEYWORDS}

NBS, NEMO, PMIPv6, Bulk Binding Update, Mobile Router.

\section{INTRODUCTION}

Internet Engineering Task Force (IETF) is standardizing various mobility management protocols, such as MIP, MIPv4, MIPv6, FMIPv6, HMIPv6, and PMIPv6. To utilize the advantages of IPv6 over IPv4 protocol, a lot of effort is directed towards improving the performance of IPv6 based protocols. One such popular MIP protocol is MIPv6 [1], which greatly reduces the handover latency compared to the MIPv4 protocol. On the other hand, NEMO basic support (NBS) [2] is adopted for mobility support in intelligent transportation systems. NBS is also a host based mobility management protocol like MIPv6. NBS is an extension of MIPv6 and allows session continuity for every node in the mobile network as the network moves. Moving network is a

Sundarapandian et al. (Eds): CoNeCo,WiMo, NLP, CRYPSIS, ICAIT, ICDIP, ITCSE, CS \& IT 07, pp. 207-223, 2012. () CS \& IT-CSCP 2012

DOI : $10.5121 /$ csit.2012.2418 
network with an internet point of attachment using MR. The MR runs with NEMO basic support protocol with Home Agent. The NBS is designed such that network mobility is transparent to the nodes inside the mobile network. So, European Telecommunications Standards Institute (ETSI) also defines the NBS based architecture support for GeoNetworking for ITS systems [3-5]. Even though it reduces the handover latency, still the delay introduced by this protocol is not acceptable to the real-time applications like VoIP. As a result, the research is directed towards the faster protocols like FMIPv6 [6]. During the last decade, the research efforts are directed towards the design and development of various faster versions of MIPv6 protocols; primarily attempting enhancement of the existing protocols.

In last decade IETF is also standardized hierarchical mobility management protocol (HMIPv6) [7], where the research is going towards study of MIPv6 protocols, HMIPv6 also contributes to performance benefits in heterogeneous wireless networks. To study the performance metrics related to mobility and traffic of various MIPv6 protocols, authors in [8], proposes a new analytical evaluation model. Performance metrics like signaling overhead cost, packet delivery cost, handoff latency and packet loss are analyzed in this work. Numerical results show that Fast handover for HMIPv6 (F-HMIPv6) improves the handover latency and packet loss than other protocols, while HMIPv6 improves the signaling overhead cost and buffer space requirement compared to other protocols.

To deploy the host based protocols like MIPv6, FMIPv6, and HMIPv6, mobile host needs to equip with the support of these protocols in their operating system. And also one of the severe drawbacks of these host based mobility management protocols is that mobile host need to be involved in the protocol operations during the handover time, which causes more signaling overhead and handover latency. So, during the year 2007-2008, the network mobility group (NEMO) from IETF standardized the network based mobility management protocol i.e. PMIPv6 [9]. Now the research is addressed towards enhancing the network based mobility management protocols and their study. A comparison between PMIPv6 and FMIPv6 is done in [10], where authors evaluated in terms of handoff latency, handoff interruption time, and expected number of dropped packets per handoff. They conclude that for faster radio access technologies in terms of handoff interruption time, FMIPv6 in predictive mode is better than MIPv6 and PMIPv6 is almost similar to reactive based FMPv6. However for slower radio access technologies PMPv6 is better than FMIPv6 in reactive mode. Because of advantages of network based mobility, PMIPv6 based mobility support is proposed even in intelligent transportation systems [11] such as in aeronautical telecommunications network. In [12], Pre-binding update scheme is proposed for seamless handover in PMIPv6 domains. This method reduces the handover latency to the very low value using proactive signaling scheme.

As next generation wireless mobile network is envisioned to be of seamless in nature, the PMIPv6 based technologies are expected to be deployed. In the PMIPv6 domain, the capacity of LMA is very high to host millions of mobility sessions. In this domain, each MAG has to send a unique binding update request for each associated mobility session even when there is no change in session state. These periodic binding update messages consume significant amount of network resources at both LMA and MAG. So it needs an optimal solution to efficiently use the network resources while performing the binding update procedures at both the entities. So the IETF has standardized an extension to PMIPv6 signaling to perform binding update/registration operations on a bulk basis using mobile node group identifier option [13]. Even though PMIPv6 provides less handover latency and signaling overhead than MIPv6 systems, the signaling over head caused by periodic binding updates for each mobility session in the PMIPv6 based networks is 
typically unacceptable in intelligent transportation systems due to their network mobility in nature. This causes a more signaling overhead which ultimately leads to inefficient network utilization. Throughout the paper the acronyms used are as given in Table 1.The rest of the paper is organized as follows: Section 2 briefly discusses the related work and section 3 discusses the proposed bulk binding approach for ITS; Section 4 contains the performance analysis and its numerical results and finally Section 5 concludes this paper.

\section{RELATED WORK}

\subsection{NBS}

A mobile network is a group of nodes connected to the internet through common gateway, known as mobile router. A mobile router has a unique Home Address (HoA) registered at the Home Agent (HA). The HoA is configured from a prefix advertised by its HA where this prefix can be advertised on the home link or delegated to the MR. MR can have more than one HoA if there are multiple prefixes in the home link. As the MR is moving away from the home link and attaches to a new access router (AR), it

Table 1. Acronyms [12]

\begin{tabular}{|l|l|}
\hline Acronym & Definition \\
\hline AAA & Authentication, Authorization, and Accounting \\
\hline AR & Access Router \\
\hline BA & Binding Acknowledgement \\
\hline BU & Binding Update \\
\hline CN & Correspondent Node \\
\hline CoA & Care-of Address \\
\hline DAD & Duplicate Address Detection \\
\hline HA & Home Agent \\
\hline HA MR $_{\text {HN }}$ & Home Agent for Mobile Router \\
\hline HNP & Home Network Prefix \\
\hline HoA & Home Address \\
\hline IETF & Internet Engineering Task Force \\
\hline ITS & Intelligent Transportation System \\
\hline LFN & Local Fixed Node \\
\hline LMA & Local Mobility Anchor \\
\hline LMA & Local Mobility Anchor for Mobile Router \\
\hline MAG & Mobile Access Gateway \\
\hline MN & Mobile Node \\
\hline MNN & Mobile Network Node \\
\hline MNP & Mobile Network Prefix \\
\hline MR & Mobile Router \\
\hline MR-ID & Mobile Router ID \\
\hline NBS & NEMO Basic Support \\
\hline NEMO & NEtwork MObility \\
\hline NS & Neighbor Solicitation \\
\hline PBA & Proxy Binding Acknowledgement \\
\hline PBU & Proxy Binding Update \\
\hline
\end{tabular}




\begin{tabular}{|l|l|}
\hline RA & Router Advertisement \\
\hline RO & Route Optimization \\
\hline RS & Router Solicitation \\
\hline BRI & Binding Revocation Indication \\
\hline BRA & Binding Revocation Advertisement \\
\hline SBA & Session to Mobility Ratio \\
\hline
\end{tabular}

obtains the Care-of Address (CoA) from the new link. As soon as the MR acquires a CoA, it sends a binding update (BU) to its HA. When the home agent receives this BU request, the HA creates a binding cache entry for the MR's home address to its Care-of address. Upon accepting $\mathrm{BU}$ request with the mobile router flag $(\mathrm{R})$ set, the HA sets up forwarding for all prefixes owned by the MR. Then the HA sends back to the MR, a binding acknowledgement (BA) with mobile router flag set.

After completing the binding update process, a bidirectional tunnel is established between the HA and the MR. The tunnel endpoints are the Home Agent's address and Mobile Router's Care-of Address. If a packet is received from the mobile network addressed to the mobile network prefix (MNP), the MR reverse-tunnels the packet to the HA through this tunnel. For the traffic originated by the MR itself, the mobile router can use either reverse tunneling or route optimization (RO). Here we considered the NBS without RO for our analysis purpose due to simplicity. The MR and HA can run a routing protocol through the bi-directional tunnel where this bidirectional tunnel must be treated as tunnel interface. The tunnel interface is included in the list of interfaces on which routing protocol is active. When MR is away from home link and connected to new link, the MR should be configured not to send any routing protocol messages on its egress interface. The signaling flow diagram for NBS based ITS system is explained in Fig. 1 where the mobile node is replaced with mobile router with group of local fixed nodes (LFN) and HA with home agent for MR $\left(\mathrm{HA}_{\mathrm{MR}}\right)$. LFN is a node connected to the gateway and does not have its own mobility capacity. 


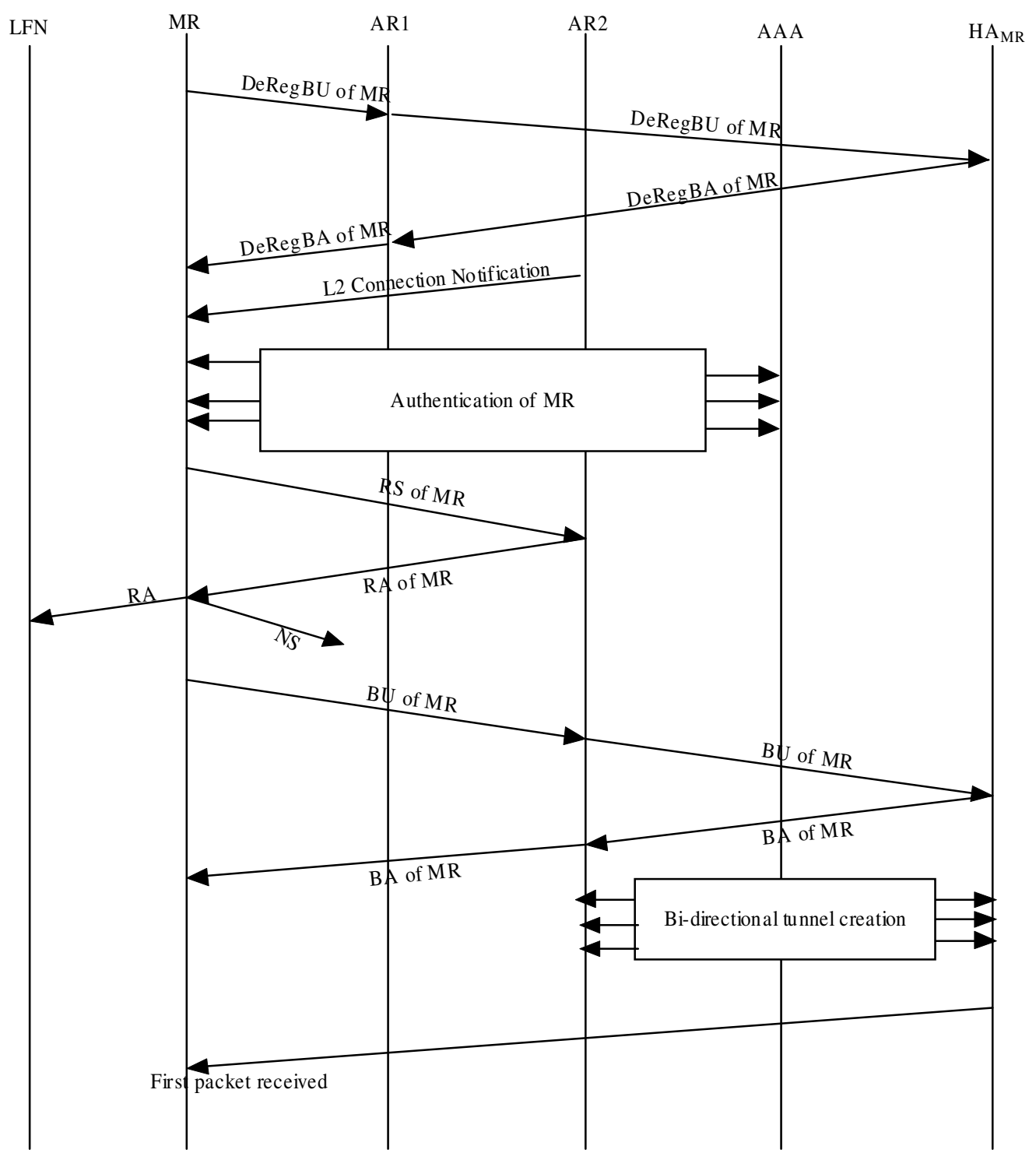

Figure 1. NEMO Basic Support (NBS)

\subsection{PMIPv6}

Mobility support in MIPv6 requires the client functionality in IPv6 stack of mobile nodes. In this regard, a lot of signaling messages need to be exchanged between the mobile node and its home agent, and also it is required to maintain the binding information between the home address and the care-off address. Network based mobility is a solution which removes the mobile node involvement in mobility signaling messages and binding update, Instead, proxy agents in networks do the mobility management on behalf of the mobile host. One such solution of network based mobility is the PMIPv6. The NEMO basic support is also being extended within PMIPv6 [14]. The signaling flow diagram of PMIPv6 during handover interruption time is explained in Fig. 2 where local mobility anchor (LMA) is represented as LMA for mobile router $\left(\mathrm{LMA}_{\mathrm{MR}}\right)$ and the mobile node is replaced with mobile router (MR) with group of local fixed nodes (LFN). 


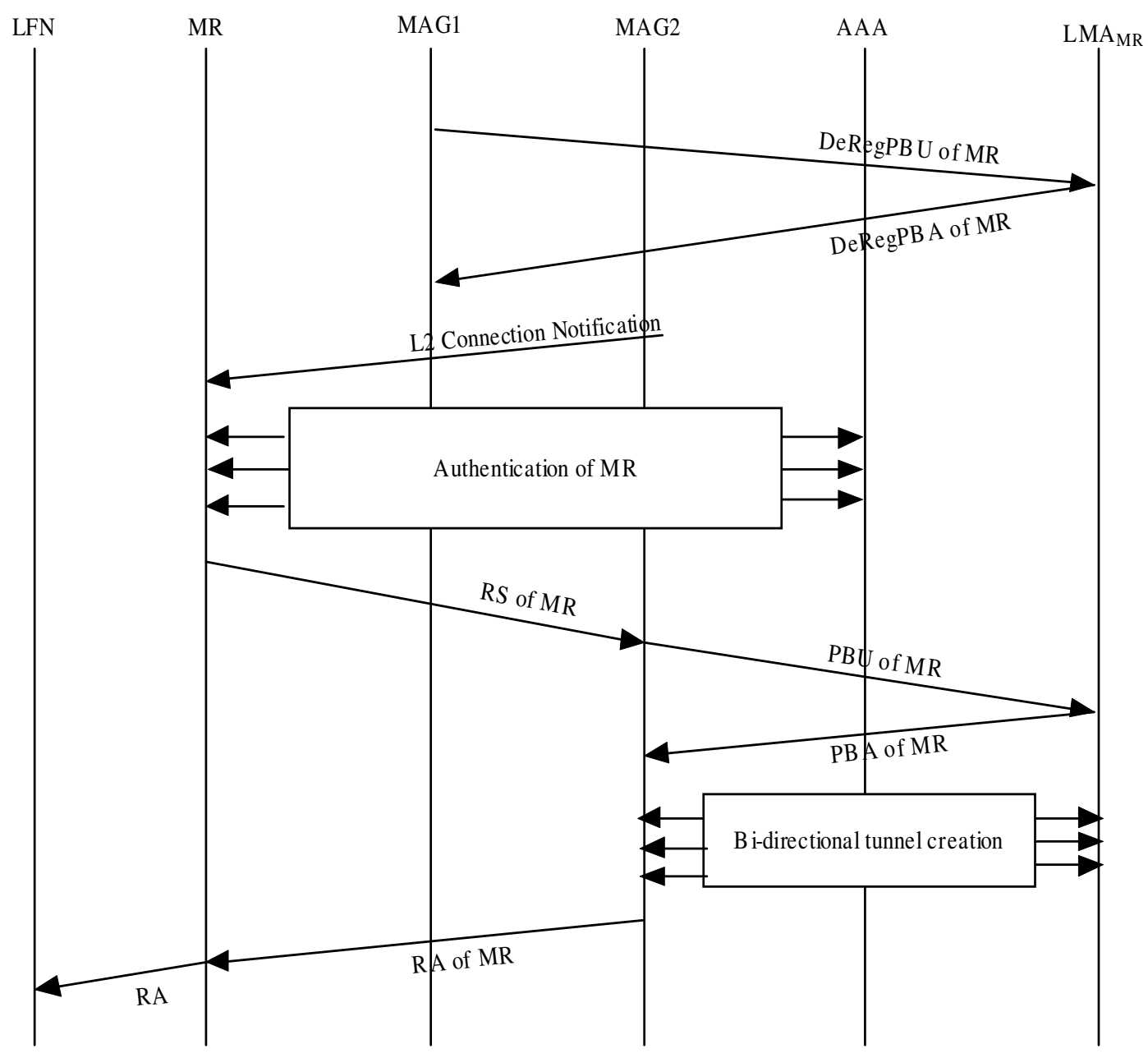

Figure 2. PMIPv6 for ITS

Two of the key components in PMIPv6 are mobile access gateway (MAG) and LMA. MAG handles all of the mobility related signaling for a mobile node that is attached to the access point, where it is also responsible for keeping track of the movements of the mobile node within the network. The functionality of LMA is similar to that of the home agent in MIPv6. It is responsible for managing the mobile node's binding state. When the mobile node enters the proxy mobile IPv6 domain, the mobile access gateway in that domain identifies the mobile node and determines whether the mobile node is authorized for the service or not [15]. Once the mobile node is authorized for network based mobility service, it can obtain home network prefixes (HNP), the default router address on that access link, and other related configuration parameters by using any of the address configuration mechanisms to move in that proxy mobile IPv6 domain. Once the router solicitation (RS) messages comes from the mobile node after attaching to the access link in a specific proxy mobile IPv6 domain, the MAG sends a proxy binding update (PBU) message to the LMA regarding the current location of the mobile node. Then the LMA sends the proxy binding acknowledgement (PBA) message, including the HNP, to the MAG.

Now, the MAG sends a router advertisement (RA) on the access link of the mobile node. After receiving the router advertisement (RA) messages, the mobile node configures its interface using 
either state-full or stateless address configuration mechanisms. After address configuration, the mobile node will be having one or more HNP at the current point of attachment to the MAG. The MAG and LMA also will be able to route the traffic through the bi-directional tunnel created as explained above.

\section{BULK BINDING UPDATE}

IETF has standardized an extension to PMIPv6 signaling to perform binding update/registration operations on a bulk basis using mobile node group identifier option [13]. There are two possible use cases of these group identifiers as follows:

1) Mobile access gateway (MAG) sends a single proxy binding update (PBU) message for a group of mobility sessions identified by a group identifier. Upon accepting the request from the MAG, the local mobility anchor (LMA) updates the lifetime of all the mobility sessions identified by the group identifier.

2) After detecting the failure of a specific service card, a LMA, or MAG can revoke all the mobility sessions by a common group identifier that are hosted on that service card.

Bulk binding update group is a group of mobility sessions that are part of the same logical group using a common group identifier. This bulk binding group is maintained by both MAG and LMA. These bulk binding update group identifiers are exchanged as part of the initial mobility session creation. The mobility entities then perform the operations related to binding update such as life time extension and revocation operations on an entire bulk binding update group.

\subsection{Operation}

While the mobile node (Mobile network with MR) is moving, the new MAG can detect it's presence on its access link, then the MAG can request the LMA to assign a bulk binding update group identifier for the mobile node's mobility session by setting the B flag in the proxy binding update (PBU) message. MAG also assigns the bulk binding update group identifier to the mobile node group identifier option. After accepting the PBU request, the LMA assigns the mobility session to a specific bulk binding update group and returns this identifier to MAG using the proxy binding acknowledge (PBA) message by setting a B flag to 1 . Once the bulk binding update group identifiers are exchanged, the LMA and the MAG can perform binding operations on these groups which means the MAG can extend the lifetime of all the mobility sessions that are part of a group by sending a single PBU message. Similarly, the LMA also can revoke all the mobility sessions that are part of a group identified by the mobile node group identifier option. The signaling flow diagram of bulk binding update based PMIPv6 during handover interruption time is explained in Fig. 3 where local mobility anchor (LMA) is represented as LMA for mobile router $\left(\mathrm{LMA}_{\mathrm{MR}}\right)$ and the mobile node is replaced with mobile router (MR) with group of local fixed nodes (LFN). Here the MR serves a network as a common gateway for the group of LFNs. The detailed operation of the bulk binding update procedure is explained in the Fig. 3 with the following steps.

Preliminary steps: 
1)As the signalling strength is going below the threshold level for MAG1 and detects more signal strength for MAG2, the MR decides to attach to MAG2. Now MAG1 detects the movement of MR1 to MAG2 and

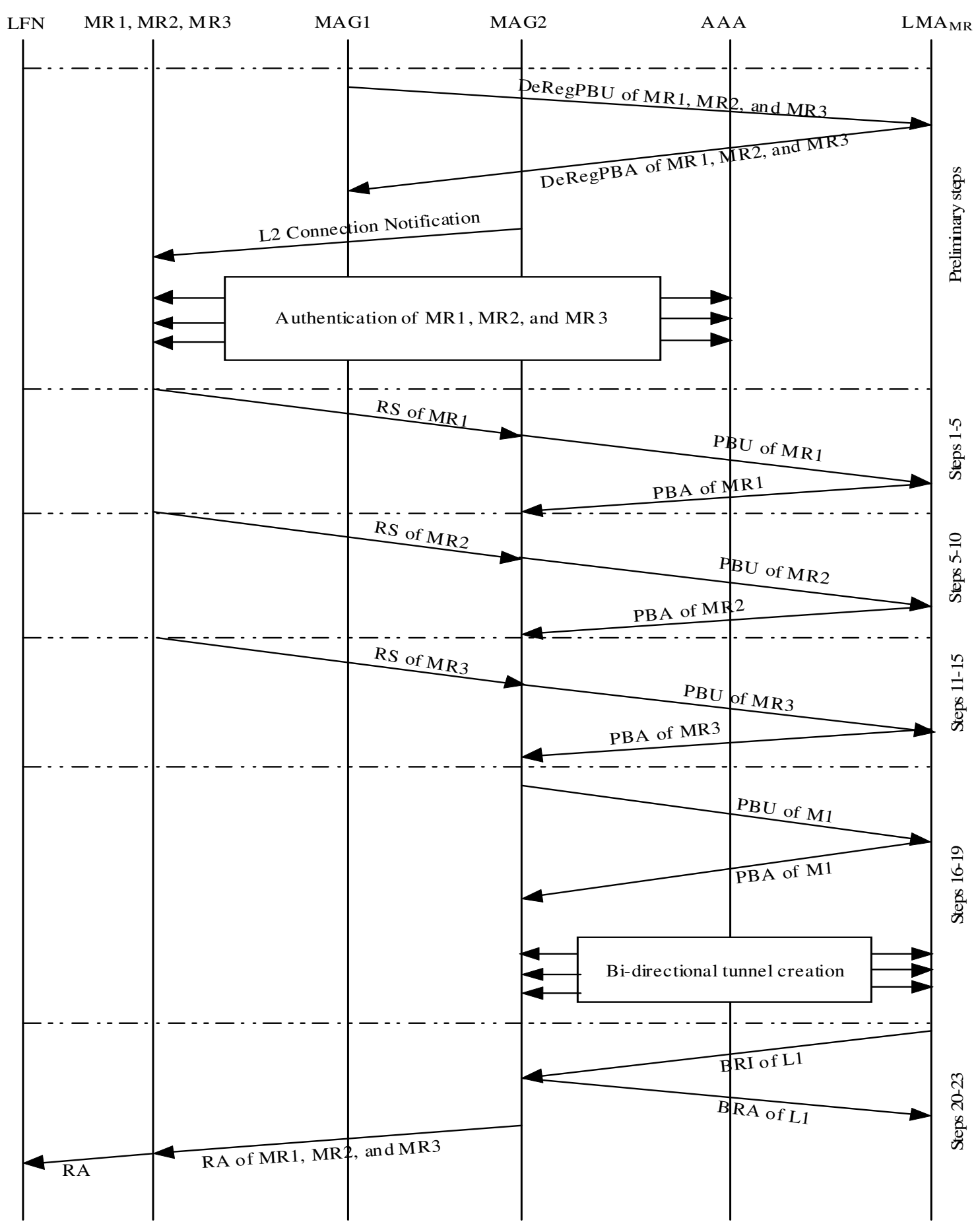

Figure 3. Bulk Binding Update Based PMIPv6 for ITS 
performs deregistration process at LMA for the MR1. Then MAG waits for the registration of MR1 through MAG2.

2) L2 handover process is performed for MR1 and then MR1 will be attached to MAG2.

3) The authentication procedure will be performed at the MAG2 for the MR1 using popular diameter/radius protocol mechanism.

These preliminary steps are also performed for the MR2, and MR3. After performing these preliminary steps for MR1, MR2, and MR3, the bulk binding update process is started at MAG2 as explained in the following steps.

Step1-2: upon detecting the attachment to the access link, the MAG assigns the MR1 to a group M1 and the group identifier is assigned by including it in the mobile node group identifier option in the PBU message.

Step3: after accepting the PBU message from MAG, the LMA creates a specific mobility session and assigns to a group L1 and sends PBA message back to MAG. LMA also updates the binding cache entry to include the bulk binding update group identifier presented by the MAG (i.e. M1) and LMA (i.e. L1).

Steps 4-5: upon receiving the PBA, the MAG also updates the binding cache entry for the mobility session by using M1, L1 identifiers. Now both MAG and LMA are aware of the group identifiers for the MR1.

Steps 6-10: Now MR2 will be participated in the bulk binding update process to exchange the group identifiers such as M1, L1. Here, the MR2 is also assigned to the same MAG's bulk binding update group M1 and LMA's bulk binding update group L1. Now MAG and LMA are also aware of MR2's mobility session group identifiers.

Steps 11-15: Now MR3 will be participated in the bulk binding update process to exchange the group identifiers such as M2, L1. Here, the MR3 is assigned to a MAG's bulk binding update group identifier M2 and LMA's bulk binding update group identifier L1. Now at this point MAG treats MR1, and MR2 as group M1 and MR3 as group M2. Whereas LMA treats the MR1, MR2, and MR3 as group L1. Now both the entities can perform the bulk binding operations on a group of mobility sessions identified by the respective bulk binding update group identifier.

Steps 16-19: the MAG sends a PBU message for extending the lifetime of all the mobility sessions identified by the group identifier M1. Upon accepting the PBU, the LMA will update the lifetimes of both MR1, and MR2 in the binding cache entries.

Steps 20-23: The LMA decides to revoke all mobility sessions that are part of the group L1. So, it sends a binding revocation indication (BRI) message with the bulk binding update group identifier (L1). Upon accepting the BRI message, the MAG revokes all the MR1, MR2, and MR3 mobility sessions, which are part of the bulk binding update group identifier L1, and sends a binding revocation acknowledgement (BRA) message. 


\section{Performance Evaluation}

Performance analysis of proposed method is done with the analytical model proposed in [11] where the authors focused mainly upon the IP-layer traffic cost and handover latency and also assumed the case of implicit mode for both protocols (NBS, and PMIPv6). We extended the performance analysis study with the proposed bulk binding update based PMIPv6 for ITS. From [11], the summary of the assumptions is given below:

1)The sessions are arriving to the mobile router (MR) in poisson distribution with a mean rate of $\lambda_{S}$ and the length of session is determined with fixed size packets.

2)It is considered M/M/1 queuing model for the packet service delay $D_{P}$, which is a sum of processing delay and transmission delay.

$D_{P}=\frac{1}{(1-\rho) \times \mu_{P}}$

Where $\rho=\frac{\lambda_{P}}{\mu_{P}}$.

3)The propagation delay for wired link $D_{\alpha}$ is considered as the ratio between physical distance between entities d and the propagation speed $\Psi$ and for wireless link $D_{\beta}$ is assumed to be $1 \mu \mathrm{s}$.

4)It is also assumed that there is no message transmission failure other than in wireless link and also wired link is robust with packet loss during the processing.

5)Link dwell time of the mobile router is assumed to be of general distribution with mean value of $\frac{1}{\mu_{L}}$. If the probability density function is given as $f_{L}^{*}(t)$, then Laplace transform is given by $f_{L}^{*}(s)=\int_{t=0}^{\infty} e^{-s t} f_{L}^{*}(t) d t$

6) For $\mathrm{N}_{\mathrm{L}}$ number of link changes during inter-session time interval, the probability that mobile router moves across $\mathrm{K}$ links is given by $\operatorname{Pr}\left[N_{L}=K\right]=\alpha(K)$. Here the $\alpha(0)=1-\frac{1-\left(f_{L}^{*}\left(\lambda_{S}\right)\right)}{S_{\sigma}}$ and $\alpha(K \geq 1)=\frac{1}{S_{\sigma}} \times\left(1-\left(f_{L}^{*}\left(\lambda_{S}\right)\right)^{2}\right) \times\left(f_{L}^{*}\left(\lambda_{S}\right)\right)^{2}$ where $S_{\sigma}$ is the session to mobility ratio (SMR) [16] and is given by $\frac{\lambda_{S}}{\mu_{L}}$.

7) Suppose $\mathrm{n}$ nodes out of total $\mathrm{N}$ are participating in bulk binding update process and these nodes are belonging to a common group, then the probability that the mobile router of this group contribute to the total signaling overhead is given by $p_{g}$. Here the $p_{g}=\frac{n}{N}$. 
The summary of notations used in analysis is given in Table 2 [11]. The signaling cost is defined as the mobility signaling overhead incurred during a handover registration. Signaling cost is denoted for protocol $\mathrm{X}$ as $C_{L U}^{X}$ and location update cost as $L U^{X}$. The packet tunneling overhead due to the proposed method is same as PMIPv6 based method proposed in [11, 17], we omitted the packet tunneling cost analysis in this paper. The signaling overhead using the bulk binding update procedure for PMIPv6 based ITS is pg times than that for PMIPv6 based ITS with the extra location update overhead of BRI, and BRA. Signaling cost is given for all the protocols including for proposed bulk binding approach as follows [11].

$$
\begin{aligned}
& C_{L U}^{N B S}=\left[\sum_{i=1}^{\infty}\left(\frac{i}{S_{\sigma}}\right) \times\left(1-\left(f_{L}^{*}\left(\lambda_{S}\right)\right)^{2}\right) \times\left(\left(f_{L}^{*}\left(\lambda_{S}\right)\right)^{i-1}\right)\right] \times L U^{N B S} \\
& L U^{N B S}=\frac{p_{f}}{1-p_{f}} \times H_{M R-A R} \times\left(S_{b u}+S_{b a}\right)+H_{A R-H A_{M R}} \times\left(S_{b u}+S_{b a}\right) \\
& C_{L U}^{P M I P v 6}=\left[\sum_{i=1}^{\infty}\left(\frac{i}{S_{\sigma}}\right) \times\left(1-\left(f_{L}^{*}\left(\lambda_{S}\right)\right)^{2}\right) \times\left(\left(f_{L}^{*}\left(\lambda_{S}\right)\right)^{i-1}\right)\right] \times L U^{P M I P v 6} \\
& L U^{P M I P v 6}=2 \times H_{M A G-L M A_{M R}} \times\left(S_{p b u}+S_{p b a}\right) \\
& C_{L U}^{P M I P v 6 \text { with Bulk Binding }}=p_{g} \times\left[\sum_{i=1}^{\infty}\left(\frac{i}{S_{\sigma}}\right) \times\left(1-\left(f_{L}^{*}\left(\lambda_{S}\right)\right)^{2}\right) \times\left(\left(f_{L}^{*}\left(\lambda_{S}\right)\right)^{i-1}\right)\right] \times L U^{P M I P v 6 \text { wih Bulk Binding }} \\
& L U^{P M I P v 6 \text { with Bulk Binding }}=2 \times H_{M A G-L M A_{M R}} \times\left(S_{p b u}+S_{p b a}\right)+H_{M A G-L M A_{M R}} \times\left(S_{b r i}+S_{b r a}\right)
\end{aligned}
$$

Handover latency is defined as the time elapses from the moment when the L2 handover starts to the moment when the mobile router receives the first packet [12]. The handover latency for a particular protocol $\mathrm{X}$ is defined as $L_{H O}^{X}[12]$ and is given as follows.

$L_{H O}^{N B S}=T_{L 2}+T_{A U}+T_{W R S}+T_{M D}+T_{D A D}+T_{L U}^{N B S}+T_{P}^{N B S}$ where, TWRS is uniformly distributed in the interval [0, MAX_RTR_SOLICITATION_DELAY]. 


$$
\begin{aligned}
& T_{M D}=T_{R S}+T_{R A} \\
& T_{R S}=T_{R A}=\frac{p_{f}}{1-p_{f}} \times H_{M R-A R} \times\left(D_{P}+D_{\beta}\right) \\
& T_{L U}^{N B S}+T_{P}^{N B S}=\frac{p_{f}}{1-p_{f}} \times H_{M R-A R} \times\left(D_{P}+D_{\beta}\right)+H_{A R-H A_{M R}} \times\left(D_{P}+D_{\alpha}\right)+\max \left(T_{B A}, T_{P}^{N B S}\right) \\
& T_{B A}=\frac{p_{f}}{1-p_{f}} \times H_{M R-A R} \times\left(D_{P}+D_{\beta}\right)+H_{A R-H A_{M R}} \times\left(D_{P}+D_{\alpha}\right) \\
& T_{P}^{N B S}=\tau \times \frac{p_{f}}{1-p_{f}} \times H_{M R-A R} \times\left(D_{P}+D_{\beta}\right)+H_{A R-H A_{M R}} \times\left(D_{P}+D_{\alpha}\right) \\
& L_{H O}^{P M I P v 6}=T_{L 2}+T_{A U}+T_{W R S}+T_{R S}+T_{L U}^{P M I P v 6}+T_{P}^{P M I P v 6} \\
& T_{R S}=\frac{p_{f}}{1-p_{f}} \times H_{M R-M A G} \times\left(D_{P}+D_{\beta}\right) \\
& T_{L U}^{P M I P v 6}+T_{P}^{P M I P v 6}=H_{M A G-L M A_{M R}} \times\left(D_{P}+D_{\alpha}\right)+\max \left(T_{P B A}, T_{P}^{P M I P v 6}\right) \\
& T_{P B A}=H_{M A G-L M A_{M R}} \times\left(D_{P}+D_{\alpha}\right) \\
& T_{P}^{P M I P v 6}=\frac{p_{f}}{1-p_{f}} \times H_{M R-M A G} \times\left(D_{P}+D_{\beta}\right)+\tau \times\left[H_{M A G-L M A_{M R}} \times\left(D_{P}+D_{\alpha}\right)\right]
\end{aligned}
$$

$L_{H O}^{P M P \vee 6 \text { Buk Binding }}=T_{L 2}+T_{A U}+T_{W R S}+T_{R S}+T_{L}^{P M M P r 6 \text { Bulk Binding }}+T_{P}^{P M M P r 6 \text { Bulk Binding }}$

$T_{L U}^{P M I P \vee 6 \text { Bulk Binding }}+T_{P}^{P M I P \vee 6 \text { Bulk Binding }}=\left(H_{M A G-L M A_{\mathcal{V R}}}+2\right) \times\left(D_{P}+D_{\alpha}\right)+\max \left(T_{P B A}, T_{P}^{P M I P \vee 6 \text { Bulk Binding }}\right)$

$$
\begin{aligned}
& T_{R S}=\frac{p_{f}}{1-p_{f}} \times H_{M R-M A G} \times\left(D_{P}+D_{\beta}\right) \\
& T_{P B A}=H_{M A G-L M A_{M R}} \times\left(D_{P}+D_{\alpha}\right) \\
& T_{P}^{P M I R v 6 \text { Bulk Binding }}=\frac{p_{f}}{1-p_{f}} \times H_{M R-M A G} \times\left(D_{P}+D_{\beta}\right)+\tau \times\left[H_{M A G-L M A_{M R}} \times\left(D_{P}+D_{\alpha}\right)\right]
\end{aligned}
$$


Parameter values used for numerical analysis are given in Table 3 [12]. A relation between signalling cost and number of link changes $\left(\mathrm{N}_{\mathrm{L}}\right)$ is shown in Fig. 4, from the Fig. 4, it has been observed that as the $\mathrm{N}_{\mathrm{L}}$ increases, the signalling cost increases linearly. Because, while the MR moves during inter-session time interval, if the number of link changes increases, the location binding update messages also increases for keeping track of MR. In NBS, the signalling overhead is effected by the wireless link failure probability $\left(p_{f}\right)$ because MR has to participate in the mobility signalling. So, in this mechanism, for $p_{f}$ of 0.5 the signalling overhead is more than that for $p_{f}$ of 0.1 as $\mathrm{N}_{\mathrm{L}}$ is varying from 1 to 8 .

Whereas, in the PMIPv6 based mechanism, the signaling overhead is same for both $p_{f}=0.1$ and 0.5. This is due to the network based mobility management where the network entities such as MAG and LMA participates in the mobility signaling on behalf of MR. However, the signaling overhead caused by the PMIPv6 based approach is higher than NBS mechanism due to double location update overhead. So, in our proposed bulk binding update method, the signaling overhead can be reduced on bulk basis registration by grouping the MRs based on their group ID. As well as, the proposed mechanism does not affected by the wireless link failure probability due to the same PMIPv6. However, as $p_{\mathrm{g}}$ increases, the signaling cost increases even worse than PMIPv6 while not effecting with $p_{f}$. As shown in Fig. 4(a), for $p_{g}$

Table 2. Notations [12]

\begin{tabular}{|c|c|}
\hline Notification & Definition \\
\hline $\mathrm{H}_{\mathrm{X}-\mathrm{Y}}$ & Average number of hops between $\mathrm{X}$ and $\mathrm{Y}$ \\
\hline $\mathrm{S}_{\mathrm{bu}}$ & Size of BU message in NBS \\
\hline $\mathrm{S}_{\mathrm{ba}}$ & Size of BA message in NBS \\
\hline$S_{\mathrm{pbu}}$ & Size of PBU message in PMIPv6 \\
\hline$S_{\text {pba }}$ & Size of PBA message in PMIPv6 \\
\hline$S_{\text {bri }}$ & Size of BRI message in bulk binding based PMIPv6 \\
\hline $\mathrm{S}_{\mathrm{bra}}$ & Size of BRI message in bulk binding based PMIPv6 \\
\hline$\tau$ & Tunneling weight factor \\
\hline $\mathrm{p}_{\mathrm{f}}$ & Wireless link failure probability \\
\hline $\mathrm{p}_{\mathrm{g}}$ & $\begin{array}{l}\text { Probability that the mobile router within group contribute to the total } \\
\text { signaling overhead }\end{array}$ \\
\hline $\mathrm{T}_{\mathrm{L} 2}$ & Link-layer handover latency \\
\hline $\mathrm{T}_{\mathrm{AU}}$ & Authentication latency \\
\hline $\mathrm{T}_{\mathrm{WRS}}$ & Random delay for sending initial RS message by the MR \\
\hline $\mathrm{T}_{\mathrm{RS}}$ & Arrival delay of RS message sent from the MR to the MAG \\
\hline $\mathrm{T}_{\mathrm{RA}}$ & Arrival delay of the RA message sent from the MAG to the MR \\
\hline $\mathrm{T}_{\mathrm{DAD}}$ & Duplicate address detection delay \\
\hline $\mathrm{T}_{\mathrm{LU}}^{\mathrm{NBS}}$ & Delay of NBS's handover registration \\
\hline $\mathrm{T}_{\mathrm{P}}^{\mathrm{NBS}}$ & Arrival delay of the first packet from $\mathrm{HA}_{\mathrm{MR}}$ to the MR \\
\hline $\mathrm{T}_{\mathrm{LU}}^{\mathrm{PMIPv6}}$ & Delay of PMIPv6's handover registration \\
\hline $\mathrm{T}^{\mathrm{PMIPv6}} \mathrm{P}$ & Arrival delay of the first packet from $\mathrm{LMA}_{\mathrm{MR}}$ to the MR \\
\hline $\mathrm{T}^{\text {PMIPv6 Bulk Binding }}{ }_{\mathrm{LU}}$ & Delay of bulk binding based PMIPv6's handover registration \\
\hline $\mathrm{T}^{\text {PMIPv6 Bulk Binding }}{ }_{\mathrm{P}}$ & $\begin{array}{l}\text { Arrival delay of the first packet from } \mathrm{LMA}_{\mathrm{MR}} \text { to the MR in the bulk } \\
\text { binding based approach }\end{array}$ \\
\hline
\end{tabular}


Table 3. Parameter values [12]

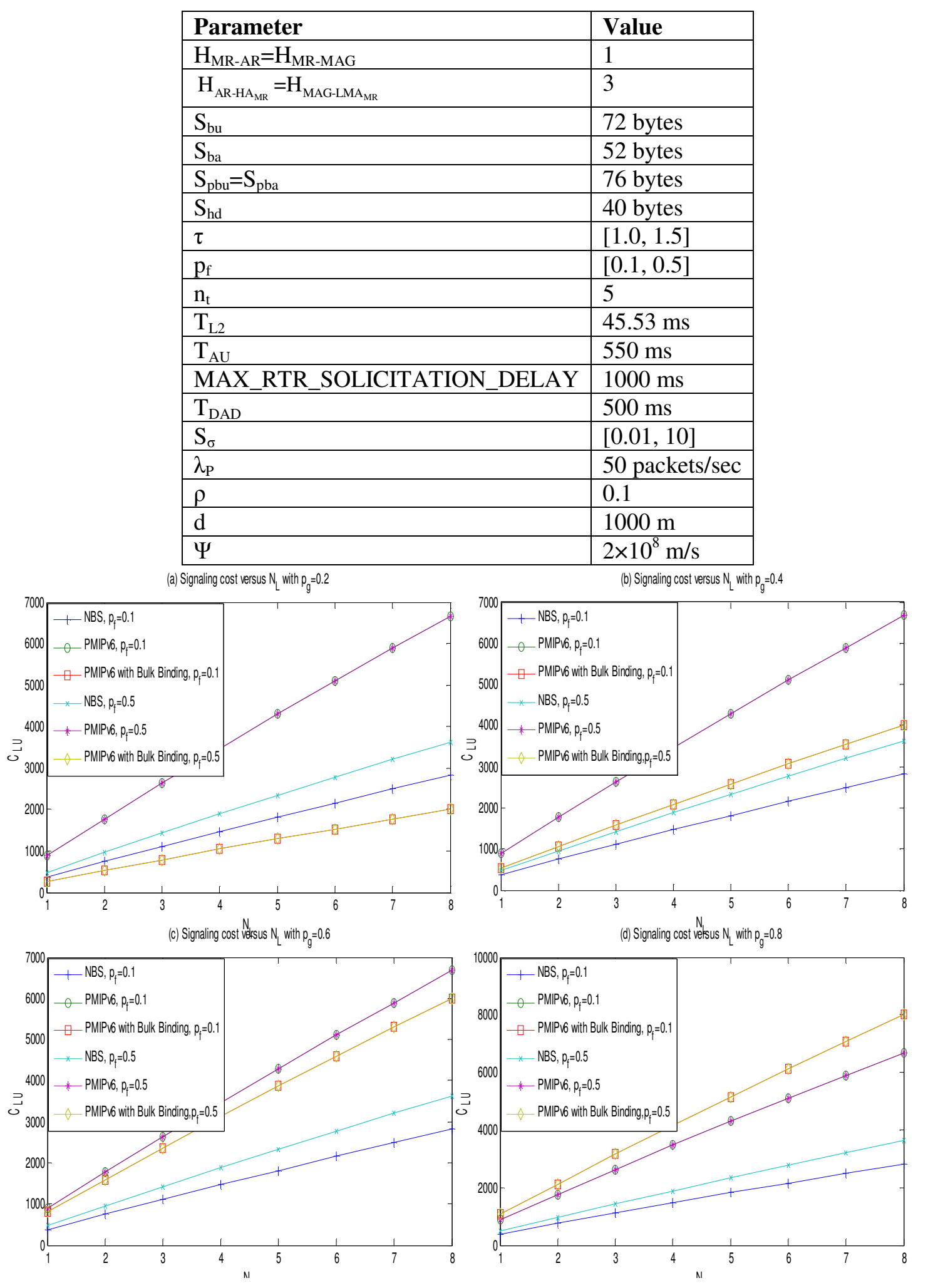


Figure 4. Signaling cost $\left(C_{L U}\right)$ versus number of link changes $\left(N_{L}\right)$ in comparison with NBS, $p_{f}=0.1$; NBS, $p_{f}=0.5$; PMIPv6, $p_{f}=0.1$; PMIPv6, $p_{f}=0.5$; Bulk Binding PMIPv6, $p_{f}=0.1$; Bulk Binding PMIPv6, $p_{f}=0.5$; (a) $p_{g}=0.2$ (b) (a) $p_{g}=0.4$ (a) $p_{g}=0.6$ (a) $p_{g}=0.8$.

of 0.2, the signaling cost of proposed approach is better than NBS, and PMIPv6. And from the Fig. 4(b) and Fig. 4(c), for $p_{g}$ of 0.4 and 0.6, the signaling cost the proposed approach is better than PMIPv6 but worse than NBS. From Fig 4(d), for $p_{g}$ of 0.8 , the proposed approach is even worse than PMIPv6. So, selecting $\mathrm{p}_{\mathrm{g}}$ is an implementation issue where the mobile routers are to be grouped in such a manner to reduce the overall signaling overhead.

From the Fig. 5(a), a handover latency performance is observed for NBS, PMIPv6, and the proposed mechanism where the proposed mechanism is almost nearly the same as PMIPv6 and less than that for NBS. This is because, in NBS, the MR has to participate in mobility management signaling. Due to this large handover delay in NBS, this method is unacceptable for real time applications. From the expanded version of Fig. 5(b), it is has been observed that the proposed approach have more handover delay than PMIPv6. However, the delay difference is in the order of micro seconds which is due to the extra processing delay occurred for the grouping involved at the mobility management entities. So, we can strongly argue that the proposed bulk binding based PMIPv6 approach for intelligent transportation systems improves the performance in terms of signaling overhead while maintaining the less handover latency.
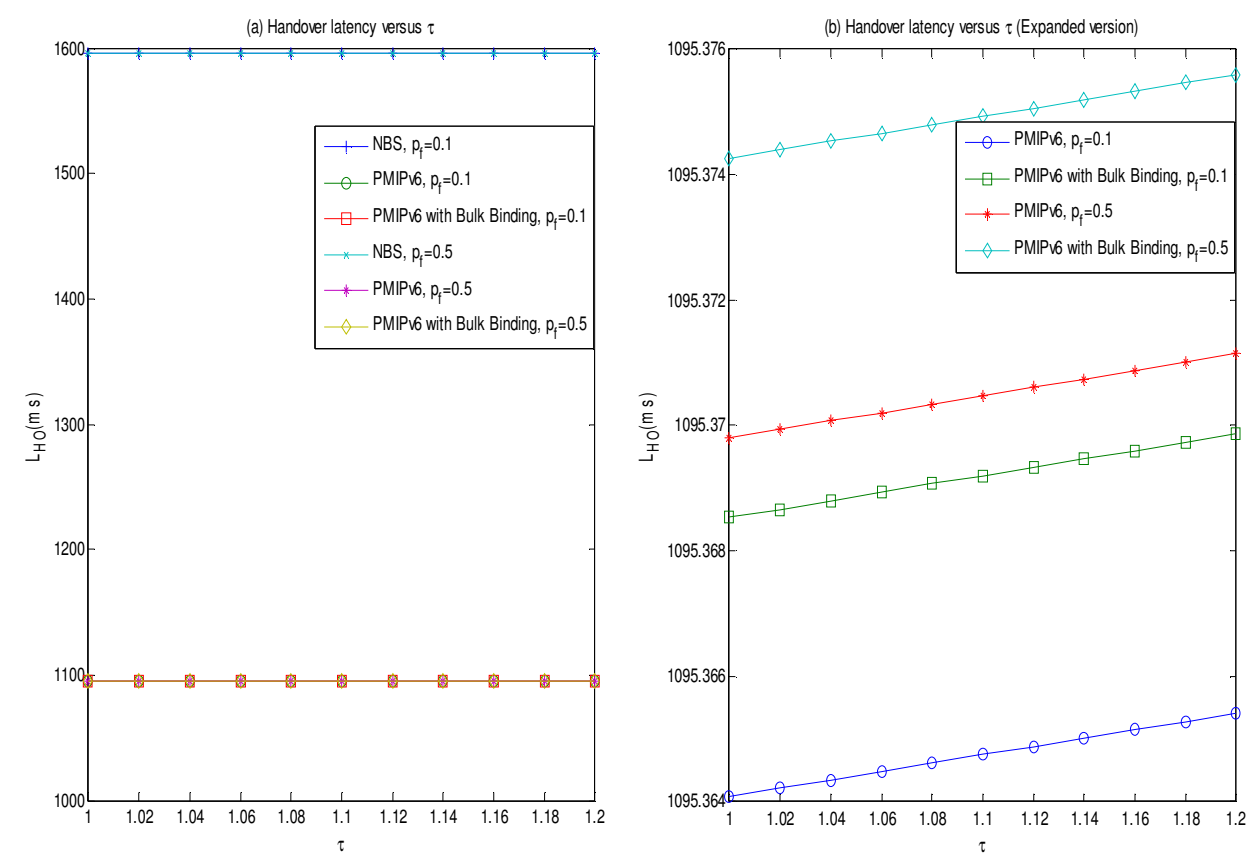

Figure 5. Handover Latency $\left(L_{H O}\right)$ versus $\tau$ in comparison with NBS, $p_{f}=0.1$; NBS, $p_{f}=0.5$; PMIPv6, $p_{f}=0.1$; PMIPv6, $p_{f}=0.5$; Bulk Binding PMIPv6, $p_{f}=0.1$; Bulk Binding PMIPv6, $p_{f}=0.5$; (a) Normal (b) Expanded. 


\section{CONCLUSION}

The NEMO basic support (NBS) is adopted as mobility management protocols for moving networks. Due to the mobile router (MR) involvement in mobility management signaling, the signaling overhead and handover latency is unacceptable resulting in high packet loss and more retransmissions. So, PMIPv6 based solutions improve the performance of handover latency than NBS by eliminating MR involvement in mobility management. However the performance of signaling overhead caused by the simple PMIPv6 based solution is unacceptable. The extension of bulk binding update procedure for simple PMIPv6 based solution for ITS is explained with the signaling flow diagrams in this paper. The conducted numerical results show the improved handover performance in terms of signaling overhead and handover latency than NBS, and simple PMIPv6 methods.

\section{ACKNOWLEDGEMENTS}

This work was carried out under the Vodafone Essar sponsored research project on Wireless Internet at IIT Kharagpur, India.

\section{REFERENCES}

[1] Johnson, C. Perkins, D. and Arkko, J., "Mobility support in ipv6", RFC 3775, June 2004.

[2] Devarapalli, V. Wakikawa, R. Petrescu, A. and Thubert, P., "Network mobility (nemo) basic support protocol", RFC 3963, Jan 2005.

[3] ETSI TS 102 636-3, Intelligent transport systems (ITS); vehicular communications; geonetworking; part 3: $\quad$ Network architecture. Version 1.1.1, Mar. 2010.

[4] ETSI TS 102 636-4-1, Intelligent transport systems (ITS); vehicular communications; geonetworking; part 4geographical addressing and forwarding for point-to-point and point-to-multipoint communications; sub-part 1: Media-independent functionality. Version 0.0.9, Nov. 2011.

[5] ETSI TS 102 636-5-1, Intelligent transport systems (ITS); vehicular communications; geonetworking; part 5: Transport protocols; sub-part 1: Basic transport protocol. Version 1.1.1, Feb. 2011.

[6] Koodli, R., "Fast handovers for mobile ipv6", RFC 4068, July 2005.

[7] Soliman, H. Castelluccia, C. Malki El, K. Bellier, and L.,"Hierarchical mobile ipv6 mobility management (hmipv6)", RFC 4140, August 2005.

[8] Makaya, C. and Pierre, S., "An analytical framework for performance evaluation of ipv6-based mobility management protocols”, Wireless Communications, IEEE Transactions on, 7(3):972 -983, March 2008.

[9] Gundavelli, S. Leung, K. Devarapalli, V. Chowdhury, K. and Patil, B., "Proxy mobile ipv6", RFC 5213, August 2008.

[10] Diab, A. and Mitschele-Thiel, A. "Comparative analysis of proxy mipv6 and fast mipv6", In Proceedings of the 7th ACM international symposium on Mobility management and wireless access, MobiWAC '09, pages 26-33, New York, NY, USA, 2009. ACM.

[11] Lee, J.-H. and Ernst, T., "Lightweight network mobility within pmipv6 for transportation systems", Systems Journal, IEEE, 5(3):352 -361, sept. 2011.

[12] Liang, Z. Jun, Z. Xuejun, Z. and Qingbo, L., "A mobility management based on proxy mipv6 and mpls in aeronautical telecommunications network", In Information Science and Engineering (ICISE), 2009 1st International Conference on, pages 2452 -2455, Dec. 2009.

[13] Abinader, F. Gundavelli, S. Leung, K. Krishnan, S. and Premec, D., "Bulk binding update support for proxy mobile ipv6", RFC 6602, May 2012.

[14] Jeon, Y. Kim, Seil. and J. Jang., "Network Mobility Support in the Proxy Mobile IPv6 Domain", Internet Draft, Draft-sijeon-mext-nemo-pmip6-00, Internet Engineering Task Force, 2010.Work in progress. 
[15] Xia, F. Sarikaya, B. Korhonen, J. Gundavelli, S. and Damic, D., "RADIUS Support for Proxy Mobile IPv6", Internet-Draft, Draft-ietf-netext-radius-pmip6-06, Internet Engineering Task Force, 2012. Work in progress.

[16] Lee, J.-H. and Chung, T.-M., "How much do we gain by introducing route optimization in proxy mobile ipv6 networks?", Annals of Telecommunications, 65:233-246, 2010. 10.1007/s12243-0090127-9.

[17] Lee, J.-H. Ernst, T. and Chung, T.-M.., "Cost analysis of ip mobility management protocols for consumer mobile devices", Consumer Electronics, IEEE Transactions on, 56(2):1010 -1017, may 2010.

\section{AUTHORS}

K Vasu is a Ph.D student at Department of Electronics and Electrical Communication Engineering at Indian Institute Technology Kharagpur, India. $\mathrm{He}$ has received his masters in Advance Networking from ABV-Indian Institute of Information Technology, Gwalior, India in 2008. His current research area is Vertical Handover in Heterogeneous Wireless Networks and Mobility Management Protocols for Next Generation Networks.

Sudipta Mahapatra graduated in Electronics and Telecommunication Engineering from Sambalpur University, Orissa, India in the year 1990. He obtained his M.Tech and Ph. D degrees in Computer Engineering from IIT, Kharagpur in the year 1992 and 1997 respectively. From April 1993 to September 2002 he was working in the Computer Science and Engineering department of National Institute of Technology, Rourkela. He was in the Electronic Systems Design Group of Loughborough University, UK, as a BOYSCAST Fellow of DST, Government of India, from March 1999 to March 2000. He joined the E \& ECE Department of IIT Kharagpur in Sept. 2002 where currently he is working as an Associate Professor. His areas of

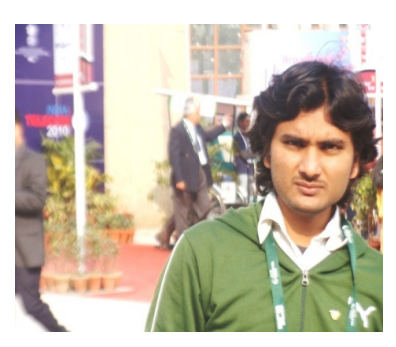
research interests include: image and video coding/compression and optical/wireless networking.

Prof C.S.Kumar received his B. Tech degree from IIT Kharagpur, West Bengal, India in 1987 and Ph.D degree from Indian Institute of Technology, Kharagpur, India in 1995.He did his P.Doc at Electro Technical Laboratory Tsukuba, Japan in 1999. Dr. Kumar is working as a Associate Professor in the Dept. of Mechanical Engineering, IIT. Kharagpur. His research activities are in ROBOTICS, INTELLIGENT SYSTEMS, COMPUTER NETWORKS, HUMAN COMPUTER INTERACTIONS
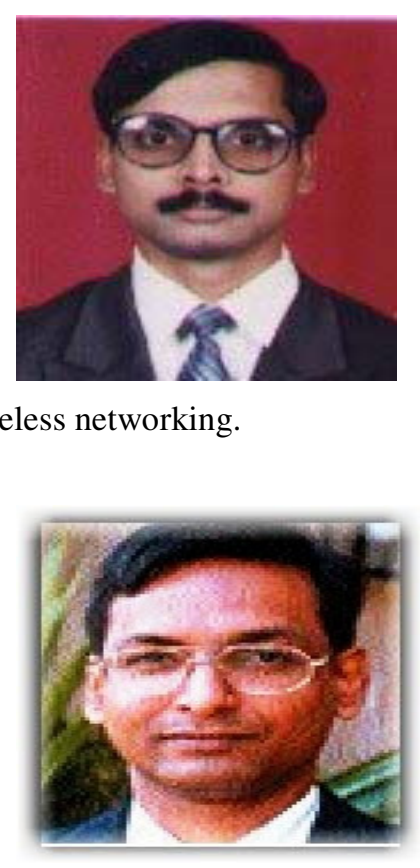